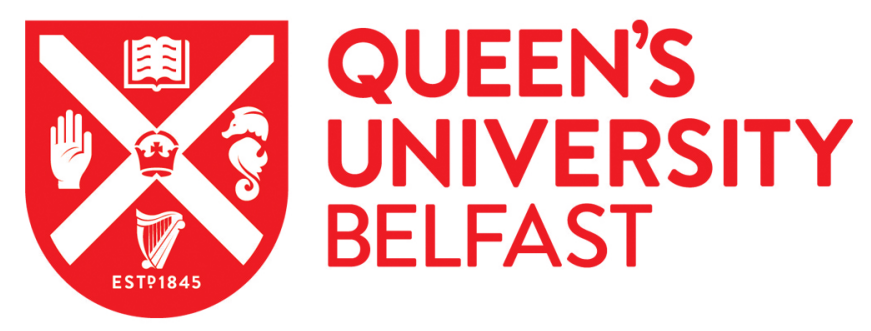

\title{
RocaSec: a standalone GUI-based package for robust co-evolutionary analysis of proteins
}

Quadeer, A. A., Morales-Jimenez, D., \& McKay, M. R. (2020). RocaSec: a standalone GUI-based package for robust co-evolutionary analysis of proteins. Bioinformatics, 36(7), 2262-2263.

https://doi.org/10.1093/bioinformatics/btz890

\section{Published in:}

Bioinformatics

Document Version:

Peer reviewed version

Queen's University Belfast - Research Portal:

Link to publication record in Queen's University Belfast Research Portal

Publisher rights

Copyright 2019 OUP. This work is made available online in accordance with the publisher's policies. Please refer to any applicable terms of use of the publisher.

\section{General rights}

Copyright for the publications made accessible via the Queen's University Belfast Research Portal is retained by the author(s) and / or other copyright owners and it is a condition of accessing these publications that users recognise and abide by the legal requirements associated with these rights.

Take down policy

The Research Portal is Queen's institutional repository that provides access to Queen's research output. Every effort has been made to ensure that content in the Research Portal does not infringe any person's rights, or applicable UK laws. If you discover content in the Research Portal that you believe breaches copyright or violates any law, please contact openaccess@qub.ac.uk. 


\title{
RocaSec: A standalone GUI-based package for robust co-evolutionary analysis of proteins
}

\author{
Ahmed A. Quadeer ${ }^{1}$, David Morales-Jimenez ${ }^{2}$ and Matthew R. McKay ${ }^{1,3, *}$ \\ 'Department of Electronic and Computer Engineering, The Hong Kong University of Science and \\ Technology, Hong Kong, China, ${ }^{2}$ Institute of Electronics, Communications and Information Technol- \\ ogy, Queen's University Belfast, Belfast, United Kingdom, ${ }^{3}$ Department of Chemical and Biological \\ Engineering, The Hong Kong University of Science and Technology, Hong Kong, China \\ ${ }^{*}$ To whom correspondence should be addressed.
}

\section{Associate Editor: $X X X X X X X$}

Received on XXXXX; revised on XXXXX; accepted on XXXXX

\begin{abstract}
Summary: Patterns of mutational correlations, learnt from protein sequences, have been shown to be informative of co-evolutionary sectors that are tightly linked to functional and/or structural properties of proteins. Previously, we developed a statistical inference method, robust co-evolutionary analysis (RoCA), to reliably predict co-evolutionary sectors of proteins, while controlling for statistical errors caused by limited data. RoCA was demonstrated on multiple viral proteins, with the inferred sectors showing close correspondences with experimentally-known biochemical domains. To facilitate seamless use of RoCA and promote more widespread application to protein data, here we present a standalone cross-platform package "RocaSec" which features an easy-to-use GUI. The package only requires the multiple sequence alignment of a protein for inferring the co-evolutionary sectors. In addition, when information on the protein biochemical domains is provided, RocaSec returns the corresponding statistical association between the inferred sectors and biochemical domains.

Availability and implementation: The RocaSec software is publicly available under the MIT License at https://github.com/ahmedaq/RocaSec.

Contact: m.mckay@ust.hk

Supplementary information: Supplementary data are available at Bioinformatics online.
\end{abstract}

\section{Introduction}

An important problem in biology is to understand how the amino acid sequence of a protein is related to its functionality and structural stability. Conventional methods to identify residues engaged in structural and functional protein domains involve laborious experiments (Berman et al., 2012; Zoller and Smith, 1982). The relatively rapid advances in sequencing technologies have inspired alternative methods based on the statistical analysis of sequence data, which have recently gained much interest.

In this regard, studying patterns of mutational correlations in the sequence data has proved useful (de Juan et al., 2013). These correlation patterns have been shown to be informative of the underlying coevolutionary sectors of residues that work together to mediate specific protein functions. Of particular note are the statistical coupling analysis (SCA) method (Halabi et al., 2009; Rivoire et al., 2016) and PCA-based methods (Dahirel et al., 2011; Quadeer et al., 2014) which have identified co-evolutionary sectors for various protein families and specific viral proteins, respectively.

A limitation of these methods was their sensitivity to statistical noise; a key issue, due to the fact that sequence data is often limited, while the number of parameters to infer is enormous. To deal with this issue, our previous work (Quadeer et al., 2018) introduced a statistical method for performing robust co-evolutionary analysis (RoCA) based on ideas from random matrix theory. This method yielded improved resilience to statistical noise. Using RoCA, we showed that specific conserved non-surface (internal) $\mathrm{HIV}$ and hepatitis $\mathrm{C}$ virus (HCV) proteins involve near-distinct and sparse sectors with modular association to protein function and structure. RoCA was also applied to other (relatively less conserved) protein families (Quadeer et al., 2018), further demonstrating its robustness and range of applicability. Here, to facilitate broadly accessible, seamless use of RoCA, we present a standalone GUI-based package "RocaSec" to robustly infer co-evolutionary sectors in conserved proteins from limited sequence data.

\section{Description of RocaSec}

RocaSec is a cross-platform package currently available for Mac, Linux, and Microsoft Windows operating systems. While implemented in MATLAB, RocaSec is distributed as a standalone application which does not require MATLAB to be installed on the user's computer. However, as the software is written in a programming language specific to MATLAB, it automatically installs the MATLAB runtime libraries necessary to run the software (only if needed) (see Supplementary Text S1 


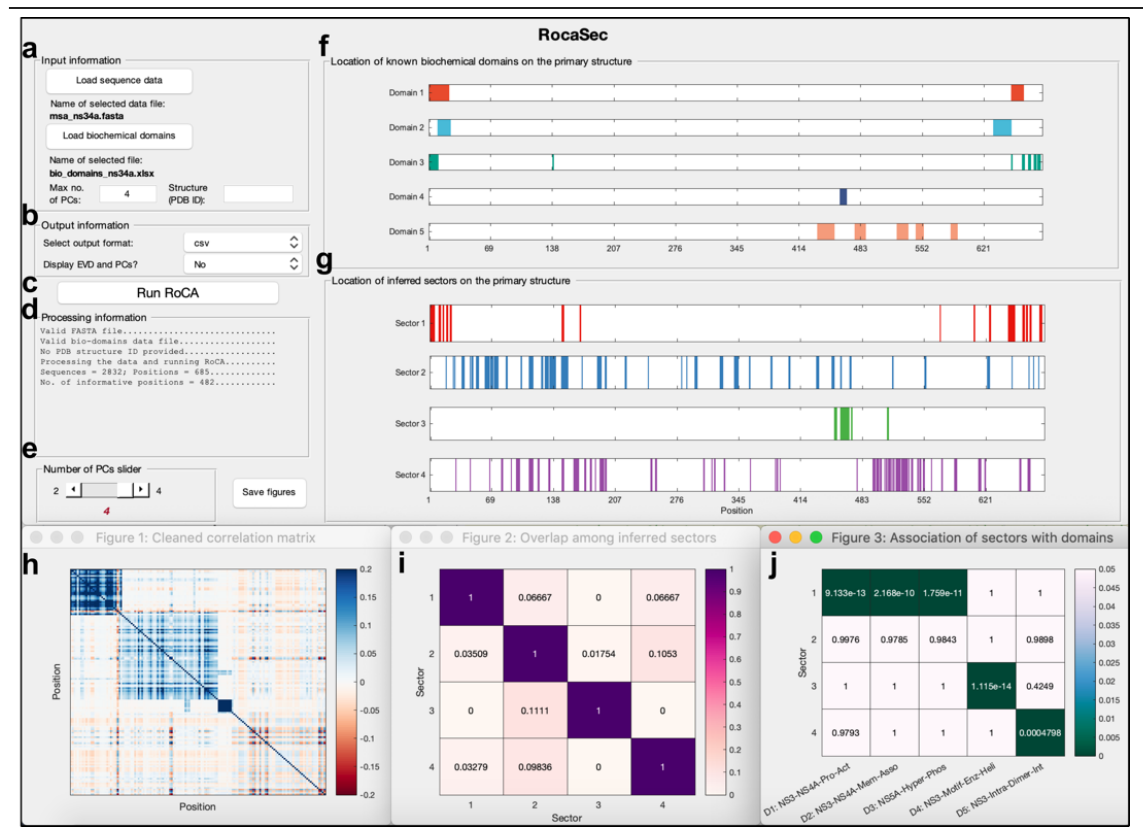

Fig. 1. The RocaSec GUI. (a) Input information required to run RoCA; (b) information about the output; (c) main execution button to run RoCA; (d) display showing information about the provided protein data and progress of RoCA execution; (e) an interactive slider for the number of PCs to visualize in real-time the different sectors inferred in each case; panel showing the location of (f) biochemical domains (if provided) and $(\mathrm{g})$ inferred sectors on the primary structure of protein; $(\mathrm{h})$ heat map of the intraand inter-sector correlations (residues that do not fall into any sector are not shown); (i) heat map of the inter-sector overlap; and (j) the statistical significance (P-value computed using Fisher's exact test) of the association of inferred sectors with the provided protein biochemical domains. Results are shown for the HCV NS3-4A protein as an example.

for details). RocaSec is featured with a user-friendly GUI with different panels where input/output options and information are conveniently displayed. In the following, we provide details of the different GUI panels and use data for HCV NS3-4A protein as a test case, reproducing results of (Quadeer et al., 2018). For results of other test HIV/HCV proteins, see Supplementary Figs. S1-S3.

Once installed, running the RocaSec application opens the GUI window (Fig. 1). The "Input information" panel (Fig. 1a) allows the user to provide the required inputs to run the RoCA method. These include: (i) the mandatory multiple sequence alignment (MSA) of the protein in FASTA format (see Supplementary Text S2); (ii) an optional input for known protein biochemical domains in xls/xlsx format (see Supplementary Text S2); (iii) the maximum number of leading principal components (PCs) to use in the inference of co-evolutionary sectors (default: 6); and an optional input that gives the user the chance to indicate the $3 \mathrm{D}$ protein structure PDB ID (available at https://www.rcsb.org).

The "Output information" panel (Fig. 1b) offers the user 2 possible format choices (either csv or xls; default: csv) to save the output files. RocaSec saves the following output files: (i) eigenvalues of the sample correlation matrix, (ii) sample PCs, (iii) RoCA estimated PCs, (iv) inferred co-evolutionary sectors (each sector produces a list of the corresponding protein residues), and (v) PyMol (a widely-used software for molecular visualization) compatible ".pml" scripts and associated mapping files (see Supplementary Text S2). The GUI also provides an option to display (in separate windows) additional statistical information, namely: the eigenvalue distribution of the sample correlation matrix and the specified PCs, including the sample PCs and the robustly estimated PCs (see e.g., Supplementary Figs. S4 and S5).

After providing input and output information, the user can click on the "Run RoCA" button (Fig. 1c) to run the RoCA method. Progress of the execution process is displayed in the "Processing information" panel (Fig. 1d) as well as in a separate progress bar which shows up while the process is running. Once the process is finished, the user can use the interactive slider for the number of PCs (Fig. 1e) to visualize in real-time the different sectors inferred in each case. The results are displayed as follows: (i) the location of residues involved in each provided biochemical domain (Fig. 1f) and in each inferred sector (Fig. 1g) is shown on the protein's primary structure, (ii) the intra- and inter-sector correlations are shown as a heat map of the cleaned correlation matrix (Quadeer et al., 2018) (Fig. 1h), and (iii) a heat map of the inter-sector overlap (Fig. 1i). The $(i, j)$-th cell of the heat map shows the overlap between sector $i$ and sector $j$, which is calculated as the fraction of residues in sector $i$ that are also in sector $j$. Additionally, if protein biochemical domains are provided (Fig. 1a), the statistical significance of the association of the inferred sectors with each domain is shown as a heat map (Fig. 1j). Finally, the user can click the "Save figures" button to save all figures as vector graphics (in eps format) for generating publication-quality figures. All the output files containing associated data (i.e., inferred sectors, sample PCs, RoCA estimated PCs, sample eigenvalues) are also saved at the same time, upon clicking the "Save figures" button

\section{Funding}

MRM and AAQ were supported by the General Research Fund of the Hong Kong Research Grants Council (RGC) (grant number 16202918).

Conflict of Interest: none declared.

\section{References}

Berman,H.M. et al. (2012) The Protein Data Bank at 40: reflecting on the past to prepare for the future. Structure, 20, 391-6.

Dahirel,V. et al. (2011) Coordinate linkage of HIV evolution reveals regions of immunological vulnerability. Proc. Natl. Acad. Sci., 108, 11530-11535.

Halabi,N. et al. (2009) Protein sectors: Evolutionary units of three-dimensional structure. Cell, 138, 774-86.

de Juan,D. et al. (2013) Emerging methods in protein co-evolution. Nat. Rev. Genet., 14, 249-61.

Quadeer,A.A. et al. (2018) Co-evolution networks of HIV/HCV are modular with direct association to structure and function. PLOS Comput. Biol., 14, e1006409.

Quadeer,A.A. et al. (2014) Statistical linkage analysis of substitutions in patientderived sequences of genotype 1a hepatitis $\mathrm{C}$ virus nonstructural protein 3 exposes targets for immunogen design. J. Virol., 88, 7628-7644.

Rivoire,O. et al. (2016) Evolution-based functional decomposition of proteins. PLoS Comput. Biol., 12, e1004817.

Zoller,M.J. and Smith,M. (1982) Oligonucleotide-directed mutagenesis using M13derived vectors: an efficient and general procedure for the production of point mutations in any fragment of DNA. Nucleic Acids Res., 10, 64876500 . 